\title{
Paroxysmal fast activity: Does this EEG pattern occur only in Lennox-Gastaut syndrome?
}

\author{
Claudia M.P. Bento ${ }^{1}$, Ana Carolina Coan ${ }^{1}$, Marilisa M. Guerreiro ${ }^{1}$ \\ ${ }^{1}$ Department of Neurology - University of Campinas (UNICAMP) - Brazil \\ Corresponding author: Marilisa M. Guerreiro; Email: mmg@fcm.unicamp.br \\ DOI: https://doi.org/10.17724/jicna.2019.151
}

Received: 23 July 2018

Accepted: 29 December 2018

\begin{abstract}
Background: Paroxysmal fast activity (PFA) is defined by fast paroxysmal events in the electroencephalogram (EEG), usually associated with Lennox-Gastaut syndrome (LGS). Our aims were to verify the frequency of LGS and non-LGS in EEGs with PFA; and to correlate the EEG features (spatial distribution, frequency, amplitude and duration) between the two clinical groups.

Methods: We analyzed 38 EEG tracings with PFA from 38 patients. We evaluated the spatial distribution, frequency, amplitude and duration of fast paroxysms. The two clinical groups (LGS and non-LGS) were statistically compared relative to the EEG data.

Results: With regard to epileptic syndromes, 23 patients (60\%) were classified as LGS and 15 patients (40\%) as non-LGS. Concerning spatial distribution, our results showed that $86.8 \%$ of the examinations showed symmetrical PFA and $13.2 \%$ showed asymmetrical PFA. The statistical analysis did not show any difference between the two groups regarding the EEG spatial distribution or other EEG data.

Conclusions: PFA can occur in other epileptic syndromes apart from LGS. The EEG features did not offer any distinction between the two clinical groups. The PFA is not a specific EEG marker of LGS.
\end{abstract}

Kewords: Cannabis, cannabinoids, cannabidiol, epilepsy, childhood.

(C) Bento CMP; licensee JICNA

\section{Background}

The paroxysmal fast activity (PFA) is characterized by paroxysmal events in the electroencephalogram (EEG) with alpha, beta and gamma frequencies ( 8 up to $>30 \mathrm{~Hz}$ ), amplitude higher than the baseline activity, lasting at least 0.2 seconds, and more frequent in the NREM sleep [1, 2, 3].

Traditionally the PFA is known as an essential feature for the diagnosis of the Lennox-Gastaut syndrome (LGS) [4]. However, it has been sporadically found in other epileptic encephalopathies and even in focal epilepsies. It was also found that their presence might be an indicator of tonic seizures and other refractory seizures, generally with poor prognosis including mental deterioration $[1,5,6,7,8,9]$.

We aimed to investigate this subject due to existing controversies on this topic and the scarcity of significant studies. Our objectives were the following: to verify the frequency of patients with LGS and patients with other epileptic syndromes (non-LGS) in EEG that presented PFA; to evaluate the EEG characteristics of PFA (spatial distribution, amplitude, frequency and duration); to compare our results between the two groups; and, to correlate the EEG data obtained from the two clinical groups (LGS and non-LGS) with the etiology (symptomatic and non-symptomatic).

\section{Methods}

We have retrieved the exams which presented the keywords "recruiting rhythm" and "paroxysmal fast activity" from our database. This research was carried out from January 2008 to April 2017 in the Electroencephalography Unit of the Clinic Hospital of UNICAMP. This is a tertiary hospital that receives mainly referral patients. Amongst the 41,269 available EEG reports, 170 exams $(0.412 \%)$ were undertaken in 45 patients, who presented at least one of their exams with PFA/recruiting rhythm. These two terms are most often described in our practice and in this study were considered as synonyms. 
The reports of the various EEGs of each patient were checked by the main author (CMPB) and, when the inclusion criterion was met, the examination was selected as a representative of the patient to be reviewed with the advisor (MMG) who has EEG credentials in our country. An average of 8.7 exams were performed per patient, ranging from 1 to 17 exams in each patient. The most recent EEG of each patient presenting PFA was reviewed in detail.

The inclusion criteria were (1) PFA per definition on EEG and (2) adequate clinical data to classify patient as LGS vs non-LGS. The PFA was characterized by paroxysmal events in the EEG with alpha, beta and gamma frequencies (8 up to $>30 \mathrm{~Hz}$ ), amplitude higher than the baseline activity, lasting at least 0.2 seconds, and more frequent in the NREM sleep $[1,2,3]$.

The PFA in EEG was classified as symmetrical or asymmetrical, according to the spatial distribution of the paroxysms (PFAs and PFAa).

It is considered PFAs when epileptiform activity is present diffusely in practically all electrodes in both hemispheres of the brain in a symmetrical way [3] (figure 1).

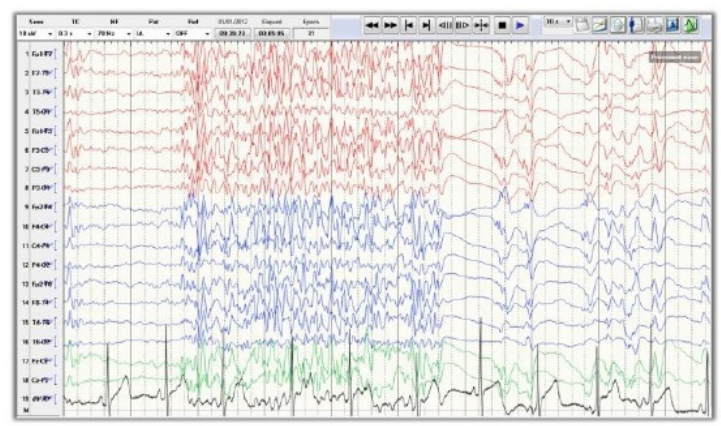

Figure 1 symmetrical PFA characterized by activity in both hemispheres of the brain symmetrically.

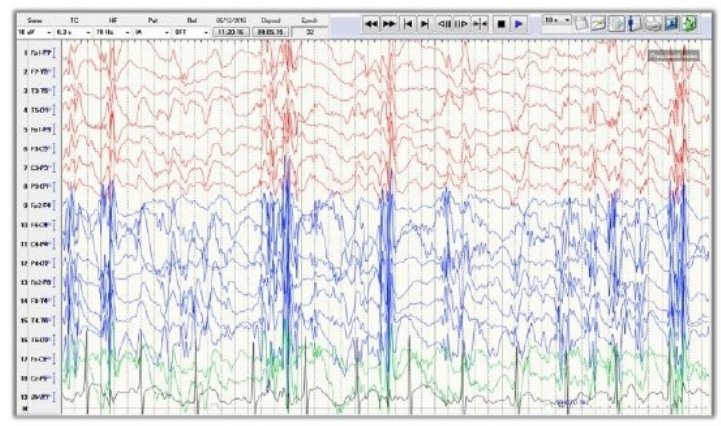

Figure 2 symmetrical PFA characterized by activity in both hemispheres of the brain symmetrically.

In the present study, we considered PFAa epileptiform activities that could not be included in the definition of PFAs. Thus, we consider asymmetrical APR with clear hemispheric predominance (Figure 2).

The systematic review of EEG allowed the quantitative analysis of the amplitude, frequency and duration of PFA.
The averages that were found were statistically analyzed.

Clinical data were collected through an interview with either the patient or the parents (or caregivers) and, subsequently, the review of the medical records to fill out a semi-structured questionnaire. The data evaluated were: age, sex, neurological diagnosis, presence, onset and frequency of the epileptic seizures, semiology of the epileptic seizures, type of the epileptic syndrome, relevant personal antecedents, neuroimaging findings and medications already used and in current use. The clinical information was collected in order to allow that patients were classified as having or not LGS. Therefore, patients were divided into two groups: patients with LGS and patients with nonLGS. The latter consisted of patients with developmental and epileptic encephalopathies (DEE) and other epileptic syndromes.

Epileptic seizures were defined according to ILAE classification $[10,11,12,13]$ and updated according to the latest ILAE publication [6]. However, the etiology has not been updated according to the new ILAE classification, since not all patients presented neuroimaging. LGS was defined according to the following triad: (a) epileptic seizures: axial tonic, atonic and atypical absence; (b) EEG abnormalities: bursts of diffuse slow spike-waves during wakefulness and bursts of fast rhythmic waves and slow polyspikes and above all generalized fast rhythms at about $10 \mathrm{~Hz}$ during sleep; and (c) a slowness in intellectual growth and associated personality disorders [4].

Patients who presented structural lesions on neuroimaging exam and/or a clear abnormal neurological examination (including cognitive deficit) were named structural cases.

The two groups (LGS and non-LGS) were compared taking into account the EEG variables and etiology of epilepsy. The spatial distribution and characteristics of the PFA regarding amplitude, frequency and duration of paroxysms were considered. A comparison between the two groups was performed using the Mann-Whitney test or the Fisher's exact test $[14,15]$. The level of significance adopted was $5 \%$. This study was approved by the Ethical Committee of our Institution (Number 1,974,229).

\section{Results}

Seven exams (out of 45 exams) were excluded, due to the fact that the reviewed exams did not present PFA with the definition adopted in this study. The remainder formed a sample of 38 patients and, therefore, 38 EEGs for final analysis $(0.09 \%$ of the total exams in the period). In the total sample of 38 patients, 71\% (27/38) were male. Regarding the classification of epileptic syndrome, 60\% (23 patients) of the patients were classified as having LGS and $40 \%$ as non-LGS subdivided into other subgroups: $37 \%$ (14 patients) as DEE and 3\% (1 patient with temporal lobe epilepsy, patient 33) as other epileptic syndromes.

The results of the EEGs enabled to observe that there are patients with symmetrical PFA (PFAs), 33 patients in to- 
tal, which corresponds to $86.8 \%$ of the tracings, and patients who had asymmetrical PFA (PFAa), 5 patients in total $(13.2 \%)$. As only 5 patients had PFAa, the test did not present statistical significance. The comparison of the two groups (LGS and non-LGS) with the spatial distribution of PFA is shown in Table 1.

Table 1 Comparison between LGS and non-LGS groups in relation to spatial distribution of PFA (Fisher's exact test)

\begin{tabular}{lcccc}
\hline & Non-LGS & LGS & Total & p-value \\
\hline PFAa & $1(6,67 \%)$ & $4(17,29 \%)$ & 5 & \\
PFs & $14(93,33 \%)$ & $19(82,61 \%)$ & 33 & \\
Total & 15 & 23 & 38 & 0.6295 \\
\hline
\end{tabular}

PFAa $=$ asymmetrical paroxysmal fast activity

PFAs $=$ symmetrical fast activity

The other EEG variables (amplitude, frequency and duration) were also analyzed in relation to both groups (LGS and non-LGS). The results are presented in Table 2 . The amplitude ranged from 15 to 758 microvolts. The frequency ranged from 8 to $62 \mathrm{~Hz}$. The duration ranged from 0.2 to 6 seconds. Thirty-six patients $(94.7 \%)$ were classified as having structural etiology, 63.9\% (23) of patients with LGS, $33.3 \%$ (12) of patients with DEE and 2.8\% (1) of patients with other epilepsy syndromes.

Table 2 Comparison between LGS and non-LGS groups in relation to spatial distribution of PFA (Fisher's exact test)

\begin{tabular}{lccccc}
\hline LGS & Variable & N & Average & Median & p-value \\
\hline Yes & Medium amplitude & 23 & 176.13 & 143.0 & 0.0944 \\
No & Medium amplitude & 15 & 232.97 & 203.0 & \\
Yes & Medium frequency & 23 & 19.71 & 18.0 & 1.00 \\
No & Medium frequency & 15 & 20.22 & 18.5 & \\
Yes & Medium duration & 23 & 1.44 & 1.00 & 0.5576 \\
No & Medium duration & 15 & 1.50 & 1.25 & \\
\hline
\end{tabular}

Structural etiology was considered when patients had abnormal neuroimaging and/or clear abnormal neurological examination, such as tetraparesis. The etiologies are shown in Table 3. The three patients considered non-structural were: one had Down syndrome (genetic) and one had Doose syndrome (probably genetic).

The other patient presented with a normal neurological examination, preserved intellectual level and normal computerized tomography scan, but no MRI. Hence, the three patients were classified as non-structural etiology.

As previously mentioned, we have also performed the comparison between the two groups considering the etiology (structural and non-structural) in relation to the spatial distribution and numerical variables. However, only three patients were non-structural, which did not allow a statistical analysis. Table 4 shows the demographic characteristics of the patients.

\section{Discussion}

In this study, we found $0.412 \%$ of all EEGs with PFA. This value differs from the data of other studies, being below the average frequency reported in the literature, which describes the PFA between $4 \%$ and up to $28 \%$ in the EEGs of tertiary centers $[2,3,6]$. We believe that this difference is due to the fact that different professionals perform the analysis and the description of the exams in our service. In addition, it is possible that the PFA has been described as polyspike activity and not as PFA or recruiting rhythm. Another factor that we have to consider is that due to the difficulty of sedation, several EEG records were carried out in EE patients only on awakeness.

Concerning epileptic syndromes, $60 \%$ of them were classified as having LGS and $40 \%$ as non-LGS. No other study has evaluated the proportion of PFA among LGS and nonLGS patients, since most studies solely addressed LGS patients $[1,6,16]$. The review of the EEGs allowed us to observe that the spatial distribution of the PFA may be symmetrical or asymmetrical (PFAs and PFAa). We have found only another study that analyzed the focality of the PFA's [3]. Therefore, we can infer that this subject is seldom addressed probably because it is not very valued.

We carried out a comparison between the groups of patients classified as having LGS versus non-LGS in relation to PFAs and PFAa. As only 5 patients presented PFAa, of whom only one non-LGS patient, the test did not present statistical significance, perhaps due to the small sample. In any case, our data suggest that symmetrical or asymmetrical EEG patterns do not allow the distinction between LGS and other epileptic syndromes.

In the quantitative analysis, we have compared the LGS versus non-LGS groups for the numerical EEG variables (amplitude, frequency and duration of PFA). This comparison neither presented statistical significance and, therefore, the EEG variables also do not allow the distinction between LGS and other epileptic syndromes. Other studies that evaluated the same numerical variables did not take into account the groups analyzed in this study. It is thus not possible to compare our findings with theirs.

It was not possible to compare the structural versus nonstructural groups in relation to the symmetrical or asymmetric PFA nor in relation to the numerical variables, since our sample did not allow the statistical analysis. The PFA does not seem to have a preference amongst patients with acquired structural, developmental or genetic etiology [3, 17].

One of our patients presented temporal lobe epilepsy with symmetrical PFA. It is possible that the PFA of this patient indicates secondary bilateral synchrony. Other studies $[2,18]$ reported that in localized epilepsies there may be PFA, and therefore, they are better called as secondary bilateral synchrony epilepsy or secondarily generalized epileptic neural network. The only difference in relation to our work is the fact that these authors pointed out that some numerical variables (duration and amplitude) are more prominent in the hemisphere ipsilateral to the lesion. In addition, 64\% 
Table 3 Comparison between LGS and non-LGS groups in relation to spatial distribution of PFA (Fisher's exact test)

\begin{tabular}{lr}
\hline Etiologies & Number of patients (percentage) $\mathbf{N = 3 6}$ \\
\hline Malformations of cortical development & $12(33.33 \%)$ \\
Hypoxic-ischemic encephalopathy & $10(27.77 \%)$ \\
Unknown cause & $5(13.88 \%)$ \\
Acquired stroke & $3(8.33 \%)$ \\
Acute anoxia due to respiratory failure (cardiac arrest and/or drowning) & $3(8.33 \%)$ \\
Meningoencephalitis & $2(5.55 \%)$ \\
Hypothalamic hamartoma & $1(2.77 \%)$ \\
Total & 36 \\
\hline
\end{tabular}

Table 4 Demographic characteristics of our patients

\begin{tabular}{|c|c|c|c|c|c|c|c|c|}
\hline Patient/Age/Gender & Age at & Age at first seizure & Perinatal insult & Neurological examination & Neuroimaging & Etiology & Structural & Lennox-Gastaut syndrome \\
\hline $1 / \mathrm{F}$ & 10 & $1 \mathrm{y} 8 \mathrm{~m}$ & No & $\mathrm{BD}$ & MRI & Tuberous sclerosis & Yes & Yes \\
\hline $2 / \mathrm{F}$ & 9 & $5 \mathrm{~m}$ & Yes & Tetraparesis + BD & MRI & CC dysgenesis & Yes & No \\
\hline 4/M & 17 & 1 day & Yes & Tetraparesis & MRI & Peri-intraventricular hemorrhage & Yes & Yes \\
\hline $5 / \mathrm{M}$ & 12 & $3 \mathrm{~m}$ & No & Tetraparesis & MRI & Unknown & Yes & No \\
\hline $6 / \mathrm{M}$ & 15 & $2 \mathrm{y}$ & Yes & Tetraparesis & CT & Unknown & Yes & Yes \\
\hline 7/M & 24 & $1 \mathrm{y} 6 \mathrm{~m}$ & No & Tetraparesis & MRI & Ito hypomelanosis & Yes & Yes \\
\hline $8 / \mathrm{M}$ & 150 & $3 \mathrm{~m}$ & No & Tetraparesis & СT & Lissencephaly & Yes & Yes \\
\hline 9/M & 13 & $1 \mathrm{y}$ & Yes & L Hemiparesis & MRI & Perinatal vascular injury & Yes & Yes \\
\hline $10 / \mathrm{M}$ & 12 & $3 y$ & No & Normal & CT & Unknown & Yes & No \\
\hline $11 / \mathrm{M}$ & 9 & $6 \mathrm{~m}$ & No & $\mathrm{R}$ hemiparesis & MRI & Acquired stroke & Yes & Yes \\
\hline $12 / \mathrm{F}$ & 15 & $3 \mathrm{~m}$ & No & Hypotonia & CT & Lissencephaly & Yes & Yes \\
\hline $13 / \mathrm{F}$ & 9 & $2 \mathrm{~m}$ & Yes & Tetraparesis & No & Unknown & Yes & Yes \\
\hline $14 / \mathrm{M}$ & 13 & $2 \mathrm{y} 8 \mathrm{~m}$ & No & $\mathrm{BD}$ & MRI & Acquired stroke & Yes & Yes \\
\hline $15 / \mathrm{M}$ & 16 & $10 \mathrm{~m}$ & No & L hemiparesis & MRI & Perinatal vascular injury & Yes & Yes \\
\hline $16 / \mathrm{M}$ & 12 & $1 \mathrm{y}$ & No & $\mathrm{R}$ hemiparesis & MRI & Hypothalamic & Yes & No \\
\hline $17 / \mathrm{F}$ & 14 & $4 \mathrm{~m}$ & Yes & $\mathrm{BD}$ & MRI & Migrational disturbance & Yes & Yes \\
\hline $18 / \mathrm{M}$ & 10 & 3days & Yes & $\mathrm{BD}$ & CT & Perinatal vascular injury & Yes & Yes \\
\hline $19 / \mathrm{F}$ & 14 & $2 \mathrm{~m}$ & No & $\mathrm{BD}$ & MRI & CC agenesis & Yes & Yes \\
\hline $20 / \mathrm{M}$ & 21 & $7 \mathrm{~m}$ & Yes & Tetraparesis + BD & CT & Unknown & Yes & Yes \\
\hline $21 / \mathrm{F}$ & 53 & $9 y$ & No & Normal & MRI & Focal cortical dysplasia & Yes & No \\
\hline $22 / \mathrm{M}$ & 20 & $7 y$ & No & Hypotonia & No & Down syndromes & No & Yes \\
\hline $23 / \mathrm{M}$ & 39 & $2 y$ & No & $\mathrm{BD}$ & MRI & Bilateral frontal lobe atrophy + Dandy-Walker complex & Yes & Yes \\
\hline $24 / \mathrm{M}$ & 21 & $9 \mathrm{~m}$ & No & L hemiparesis & MRI & $\mathrm{R}$ hemimegalencephaly & Yes & No \\
\hline $25 / \mathrm{F}$ & 5 & $1 \mathrm{y} 6 \mathrm{~m}$ & No & Tetraparesis & No & Meningoencephalitis & Yes & Yes \\
\hline $26 / \mathrm{M}$ & 16 & $2 \mathrm{y} 6 \mathrm{~m}$ & No & Intellectual deficiency & MRI & Doose syndrome & No & No \\
\hline
\end{tabular}

of their patients became seizure-free after surgical resection. Therefore, the PFA may be present in patients with a structural cause of epilepsy with secondary bilateral synchrony and its presence does not always mean a poor prognosis [2].

\section{Conclusion}

Although the PFA is an important diagnostic feature of the LGS, our study has clearly demonstrated that the PFA can also occur in other epileptic syndromes. The EEG characteristics (symmetrical versus asymmetrical) as well as the numerical variables (duration, amplitude and frequency) of the PFA do not differentiate LGS from other epileptic syndromes. Finally, the small sample did not allow statistical correlation when the etiology was considered.

\section{Aknowledgments}

Dr. Claudia M. P. Bento received a scholarship from the National Council for Scientific and Technological Development (CNPq) (Grant No 159593/2015-8).

\section{Competing interests}

The authors have declared that they have no competing interests.

\section{Author contributions}

All the authors contributed to data collection and also critically reviewed the manuscript. The final version of the manuscript was approved by all the authors.

This is an Open Access article distributed under the terms of the Creative Commons Attribution License (http://creativecommons.org/licenses/by/4.0), which permits unrestricted use, distribution, and reproduction in any medium, provided the original work is properly credited. The Creative Commons Public Domain Dedication waiver (http://creativecommons.org/publicdomain/zero/1.0/)

applies to the data made available in this article, unless otherwise stated.

Cite this article as:

Bento, C. M., Coan, A. C., \& Guerreiro, M. M. (2019). Paroxysmal fast activity: Does this EEG pattern occur only in Lennox-Gastaut syndrome?. Journal of the International Child Neurology Association, 1(1). https://doi.org/10.17724/jicna.2019.151

\section{References}

[1] Bourgeois BFD, Douglass LM, Sankar R. LennoxGastaut syndrome: A consensus approach to differential diagnosis. Epilepsia. 2014;55(s4):4-9. PubMed. 
[2] Mohammadi M, Okanishi T, Okanari K, Baba S, Sumiyoshi H, Sakuma S, et al. Asymmetrical generalized paroxysmal fast activities in children with intractable localization-related epilepsy. Brain and Development. 2015 Jan;37(1):59-65. PubMed.

[3] Wu JY, Koh S, Sankar R, Mathern GW. Paroxysmal fast activity: An interictal scalp EEG marker of epileptogenesis in children. Epilepsy Research. 2008;82(1):99 106. PubMed.

[4] Crespel A, Gelisse P, Nikanorova M, Ferlazzo E, Genton P. Lennox-Gastaut syndrome. Epileptic syndromes in infancy, childhood and adolescence. 2012;5:189-216.

[5] Arzimanoglou FJBWTCJHEJPFMGPGRKGPJMPEW Alexis, W J. Lennox-Gastaut syndrome: a consensus approach on diagnosis, assessment, management, and trial methodology. The Lancet Neurology. 2009 Jan;8(1):82-93. PubMed.

[6] HALÁSZ P, JANSZKY J, BARCS G, SZUCS A. Generalised paroxysmal fast activity (GPFA) is not always a sign of malignant epileptic encephalopathy. Seizure - European Journal of Epilepsy. 2004 Jun;13(4):270276. PubMed.

[7] Fisher RS, Cross JH, French JA, Higurashi N, Hirsch E, Jansen FE, et al. Operational classification of seizure types by the International League Against Epilepsy: Position Paper of the ILAE Commission for Classification and Terminology. Epilepsia. 2017;58(4):522530. PubMed.

[8] Pillay N, Archer JS, Badawy RAB, Flanagan DF, Berkovic SF, Jackson G. Networks underlying paroxysmal fast activity and slow spike and wave in LennoxGastaut syndrome. Neurology. 2013;81(7):665-673. PubMed.

[9] Piña-Garza JE, Chung S, Montouris GD, Radtke RA, Resnick T, Wechsler RT. Challenges in identifying Lennox-Gastaut syndrome in adults: A case series illustrating its changing nature. Epilepsy \& Behavior Case Reports. 2016;5:38 - 43. PubMed.

[10] Berg AT, Berkovic SF, Brodie MJ, Buchhalter J, Cross $\mathrm{JH}$, Van Emde Boas W, et al. Revised terminology and concepts for organization of seizures and epilepsies: Report of the ILAE Commission on Classification and Terminology, 2005-2009. Epilepsia. 2010;51(4):676685. PubMed.

[11] Berg AT, Scheffer IE. New concepts in classification of the epilepsies: Entering the 21st century. Epilepsia. 2011;52(6):1058-1062. PubMed.

[12] Engel Jr J. A Proposed Diagnostic Scheme for People with Epileptic Seizures and with Epilepsy: Report of the ILAE Task Force on Classification and Terminology. Epilepsia. 2001;42(6):796-803. PubMed.
[13] Engel Jr J. Report of the ILAE Classification Core Group. Epilepsia. 2006;47(9):1558-1568. PubMed.

[14] Conover WJ. Practical Nonparametric Statistics, 3rd edition. Scottsdale, AZ, U.S.A.: John Wiley \& Sons; 1999.

[15] Fleiss JL. Statistical Methods for Rates and Proportions, 1st Edition. New York, NY, U.S.A.: John Wiley \& Sons; 1981.

[16] Ferlazzo E, Nikaronova M, Italiano D, Bureau $M$, Dravet C, Calarese T, et al. Lennox-Gastaut syndrome in adulthood: Clinical and EEG features. Epilepsy Research. 2010;89(2):271 - 277. PubMed.

[17] Sueda K, Takeuchi F, Shiraishi H, Nakane S, Sakurai $\mathrm{K}$, Yagyu K, et al. Magnetoencephalographic analysis of paroxysmal fast activity in patients with epileptic spasms. Epilepsy Research. 2013;104(1):68 - 77. PubMed.

[18] Archer JS, Warren AEL, Stagnitti MR, Masterton RAJ, Abbott DF, Jackson GD. Lennox-Gastaut syndrome and phenotype: Secondary network epilepsies. Epilepsia. 2014;55(8):1245-1254. PubMed. 